\title{
EVOLUCIÓN METODOLÓGICA DE LA ENSEÑANZA DEL ÁRABE MARROQUÍ EN ESPAÑA: \\ DEL MODELO TRADICIONAL A LOS ENFOQUES COMUNICATIVOS
}

\author{
Rosa SALGADO SUÁREZ \\ Universidad de Sevilla
}

BIBLID [1133-8571] 26 (2019) 15.1-22.

Resumen: La historia de la enseñanza formal del árabe marroquí en España se remonta a finales del siglo XVIII y continúa hasta la actualidad. Durante estos poco más de dos siglos su enseñanza se ha ido adaptando a los métodos, hoy llamados enfoques, más afamados en toda Europa para la enseñanza de lenguas vivas. El objeto de este artículo es analizar la evolución metodológica del árabe marroquí en España para comprender mejor los desafíos que, en torno a esta disciplina, se plantean en el futuro.

Palabras clave: Árabe marroquí, Dāriya, Métodos de enseñanza.

Abstract: «Methodological evolution of teaching Moroccan Arabic in Spain: from Traditional Model to Communicative Approach ». The history of Moroccan Arabic Studies in Spain dates back to the late eighteenth century and continues to the present day. During these two centuries, teaching Moroccan Arabic has been adapted to the most famous methods used in Europe for teaching modern languages. The goal of this communication is to analyze the methodological developments in Moroccan Arabic education to better understand the challenges that will arise in the future.

Key words: Moroccan Arabic, Dāriya, Teaching methods.

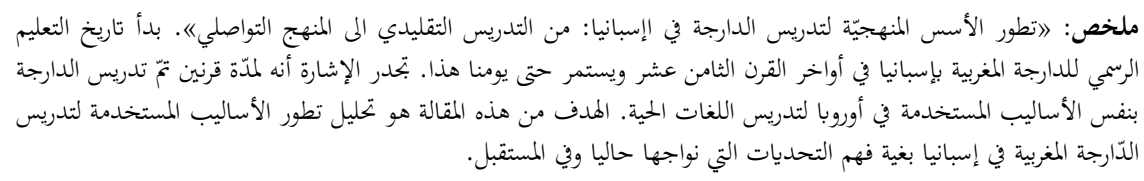

* Profesora sustituta interina. E-mail: rsalgado@us.es. ORCID: 0000-0002-7084-4669. 
كلمات مفاتيح: الدّارجة المغربية، الدّارجة، أساليب التدريس.

\section{Introducción}

La historia de la enseñanza formal del árabe marroquí en España se remonta a finales del siglo XVIII y continúa hasta la actualidad (Aguilar y Bouhrass 2010: 163-185). Durante estos poco más de dos siglos la enseñanza del árabe marroquí se ha ido adaptando, con sus especificidades, a los métodos -hoy llamados enfoques- más afamados en toda Europa para la enseñanza de lenguas vivas (Arias \& Peña 1995: 11-38). Así se intentó dar respuesta, por una parte, a las demandas y exigencias del africanismo español antes y durante el Protectorado de España en Marruecos desde finales del siglo XVIII hasta mediados del siglo XX y, por otra, al fenómeno de la inmigración y cuestiones académicas desde finales del siglo XX hasta nuestros días. Los distintos métodos aplicados en la enseñanza del árabe marroquí se han ido concretando en torno a más de cuarenta manuales ${ }^{(1)}$ de árabe marroquí de muy diverso tipo, como gramáticas, métodos, guías o cartillas, publicados en español entre 1807 y 2013 (Gómez 1995: 18-23), aunque, solo cuatro de ellos son susceptibles de ser utilizados hoy por estar alejados ya de un discurso colonial y por estar adaptados a un enfoque comunicativo.

Desde el siglo XVIII hasta bien entrado el siglo XX, la poca implicación de los arabistas del ámbito universitario en la enseñanza del árabe marroquí, considerado un árabe «vulgar» y «corrupto» mientras que el árabe clásico era tenido como la lengua «perfecta» y «suprema», no solo en términos lingüísticos sino también religiosos, y el pesado lastre que la enseñanza gramatical ha supuesto en la enseñanza del árabe marroquí debido a un error en el punto de partida como es la concepción de la lengua y la conciencia lingüística (Aguilar y Bouhrass 2010: 174-175) condicionó, en buena medida, su enseñanza y

(1) En este artículo se entiende por «manuales»: apéndices y obras monográficas con carácter didáctico publicados en español en los que sus autores aplicaron un «método o enfoque» para presentar los contenidos susceptibles de ser enseñados, en función de sus ideas sobre la naturaleza del árabe marroquí, su enseñanza y aprendizaje y el contexto en el que esta iba a tener lugar, en cada momento histórico. No se han incluido en esta investigación manuales manuscritos y/o sin publicar ni otros materiales complementarios como glosarios, silabarios, diccionarios o textos aunque todos ellos contribuyen igualmente al campo de la enseñanza del árabe marroquí en España. 
aprendizaje. En consecuencia, la enseñanza del árabe marroquí se hizo extramuros del ámbito universitario y quienes ejercieron como docentes o «manualistas» no siempre fueron arabistas (Salgado 2017: 2623-2637) sino militares, religiosos o intérpretes que habían aprendido el árabe marroquí por circunstancias biográficas o por haber realizado estancias más o menos breves en el, por entonces, lejano Marruecos (Arias 1995: 321-336).

Desde finales del siglo XX, sin embargo, la enseñanza del árabe marroquí ha ido experimentando cambios relevantes gracias a su inclusión en lo que podríamos denominar el «ámbito científico» (Sánchez 2009: 65). La publicación de nuevas investigaciones desde el ámbito académico ha contribuido a renovar la conciencia lingüística sobre el árabe marroquí y las discusiones sobre su dimensión pedagógica y pragmática. Esto ha repercutido en la inclusión del árabe marroquí en las universidades y en otras instituciones españolas desde finales del siglo XX y en una enseñanza más comunicativa que se ha ido ajustando a los principios del Marco Común Europeo de Referencia para las Lenguas (MCER 2001) y concretando en nuevos manuales de árabe marroquí para un público hispanohablante en el transcurso del siglo XXI.

No obstante, todavía queda mucho camino por recorrer en el campo de la enseñanza y el aprendizaje del árabe marroquí en nuestro país. Por ello, mostrar la evolución metodológica de esta disciplina desde finales del siglo XVIII hasta la actualidad para reflexionar sobre los retos que la enseñanza del árabe marroquí plantea en el futuro es el objeto de este artículo.

\section{Los inicios de la enseñanza del árabe marroquí en España a finales del siglo XVIII}

La historia de la enseñanza formal del árabe marroquí en España se inicia en una primera etapa que abarca desde finales del siglo XVIII hasta la llegada de la Guerra de la Independencia de 1808. Por entonces, como bien ha señalado Ángeles Vicente (2008: 20-23), ya se habría producido un evento histórico de singular importancia, como fue la llegada de Napoleón a Egipto, que simboliza el contacto entre el mundo árabe y Europa. Este hecho puso en contacto a militares, religiosos, viajeros, cuerpo diplomático y consular o filólogos, estos últimos expertos en el árabe clásico para la comprensión de textos, con distintas variedades vernáculas árabes, como el árabe marroquí, haciendo surgir los primeros centros y manuales para su enseñanza y aprendizaje por razones imperialistas, religiosas o filológicas. 
En el caso concreto de España, fue la firma del Canónico Tratado de Amistad y Comercio de 1767 (Feria 2005: 3-26) entre el Borbón Carlos III y el sultán de Marruecos, el acontecimiento histórico que suscitó el interés por el árabe marroquí por razones diplomáticas y mercantiles. La firma del Tratado puso de manifiesto la escasez de traductores e intérpretes de los que disponía España para mediar con Marruecos tras el eclipse que habían sufrido los estudios árabes en el siglo XVII. Fue el Borbón Carlos IV quien puso en marcha dos importantes medidas, a remolque de las ya iniciadas por Carlos III, el impulsor de cátedras de árabe en España, para formar una improvisada plantilla de traductores e intérpretes de confianza que dieron inicio, también, a la historia de la enseñanza formal del árabe marroquí: la primera medida fue enviar a arabistas españoles de la talla del jerónimo fray Patricio de la Torre, catedrático de árabe en el Colegio de San Lorenzo (El Escorial), y a dos agregados laicos, Manuel Bacas Merino y Juan de Arce y Morís, a Marruecos por R. O. de 1798 y la segunda medida fue crear una escuela de árabe en Tánger en 1800 para la formación lingüística de franciscanos (Justel 1991). Se formaba así la primera generación de expertos españoles en el árabe marroquí. Y entre ellos, el arabista Manuel Bacas Merino, se convertía en el autor de la primera gramática de árabe marroquí publicada en español (Moscoso 2008: 269-293).

\subsection{El Modelo Gramatical}

Cuando Manuel Bacas Merino compuso su Compendio Gramatical para aprender la lengua arábiga así sabia como vulgar (1807) se basó en el Modelo Tradicional de herencia grecolatina y árabe, en su corriente gramatical, configurado a partir de dos tradiciones bien asentadas ya en el tiempo y que tenían como manual privilegiado las obras de gramática. La tradición gramatical grecolatina y árabe se habrían puesto ya juntas desde al menos el siglo XVI cuando el jerónimo toledano Fray Pedro de Alcalá publicó el Arte para ligeramente saber la lengua arauiga (1505), una gramática que atendía al árabe granadino y que se organizaba según las gramáticas latinas pero que incluía, también, conceptos de la tradición gramatical árabe (Zwartjes 1993: 262). No obstante, fue un siglo más tarde, cuando ambas tradiciones se concretaron en la Grammatica Arabica (1613) del orientalista holandés Thomas Van Erpe, una gramática pedagógica para el estudio del árabe clásico que permitió «gramatizar» el árabe en el Occidente moderno, no solo a partir del modelo latino sino también de la tradición gramatical árabe (Girard 2013: 16-19). Bajo 
los principios de este modelo de enseñanza, Bacas Merino entendió el árabe marroquí como un conjunto de reglas gramaticales que debían aprenderse memorizando a partir de los moldes del árabe clásico. Por ello, este autor elaboró una gramática contrastiva árabe clásico - árabe marroquí tomando como referencia la gramática de Erpenio que siguió siendo el manual más afamado para el aprendizaje del árabe clásico en Europa hasta bien entrado el siglo XIX. El Compendio, contenía una tabla de transcripción figurada, muy sui generis, como todas las que vendrían después, enunciados gramaticales en español dispuestos según el orden de la oración árabe (el nombre, el verbo, la partícula) y algunos ejemplos ilustrativos escritos en árabe clásico, en árabe marroquí o transcripción figurada. Merino lograba así «gramatizar» el árabe marroquí en español para facilitar su aprendizaje a los españoles encargados de las relaciones políticas y mercantiles con Marruecos (Bacas Merino 1807: V-XIII).

\section{La regularización de la enseñanza del árabe marroquí en vísperas del Protectorado de España en Marruecos}

En el transcurso del siglo XIX, nuevos acontecimientos en el escenario europeo hicieron resurgir el interés por el árabe marroquí en España, tras el paréntesis que esta actividad había sufrido debido a la Guerra de la Independencia. Así se inició una segunda etapa que se prolongó hasta la instauración del Protectorado de España en Marruecos (1912). En efecto, la colonización francesa de Argelia (1830) o la Batalla de Isly entre Francia y Marruecos (1844), fueron algunos de los acontecimientos que vinieron a alterar el statu quo imperante entre España y Marruecos. España se unió entonces a un juego de alianzas con las potencias europeas para conquistar Marruecos en virtud de los lazos históricos que le unían con el país vecino desde tiempos de los Reyes Católicos. Surgía entonces en España una corriente de opinión favorable a la penetración militar y otra a favor de una intervención diplomática, política y económica en Marruecos que culminaron con la instauración de un doble Protectorado franco-español en 1912.

En este contexto, el movimiento africanista español, contemporáneo al surgido en Europa en el último tercio del siglo XIX, se interesó por el árabe marroquí, mientras que Francia lo hacía por el árabe argelino (Larzul 2013: 5478). Los africanistas españoles entendieron la cuestión del árabe marroquí como un pilar más del africanismo español para poder comprender mejor a aquellos a quienes se pretendía conquistar. El árabe marroquí debía, pues, estar en boca no 
solo de la plantilla de la Carrera de Intérpretes del Estado sino también en boca de todos aquellos dispuestos a conquistar aquella «presa codiciada» que era Marruecos. Y así fue como, gracias a iniciativas militares, religiosas, mercantiles y académicas, se regularizaba en España la enseñanza del árabe marroquí, de nuevo, extra-muros del ámbito universitario. Su enseñanza se difundió desde la Escuela de Árabe de Tetuán, las Academias Militares de Ceuta y Melilla, los Centros Comerciales Hispano-marroquíes, las Escuelas Superiores de Comercio o el Instituto Libre de Enseñanza y el Centro de Estudios Marroquíes. Se publicaron, también, doce manuales de árabe marroquí gracias, especialmente, a la labor de militares y, en menor medida, de religiosos, intérpretes o arabistas. Estos manuales se destinaron a africanistas de diversos ámbitos para que mediante la enseñanza formal o el aprendizaje autodidacta pudieran conocer no solo la lengua sino también la cultura de los marroquíes.

\subsection{El Método de Gramática y Traducción y el Modelo Conversacional}

La enseñanza de variedades vernáculas árabes como el argelino o el marroquí se adaptaron en el transcurso del siglo XIX a las novedades metodológicas de la Europa del siglo XIX que, gracias a los avances en la lingüística, el comparativismo y el racionalismo, dieron un toque más práctico a la enseñanza gramatical para adaptarse a las necesidades de comunicación entre los países en los que, además, aumentaban los centros para la enseñanza de lenguas extranjeras (Sánchez 1992: 137-171).

En España, la enseñanza del árabe marroquí en vísperas del Protectorado se siguió adaptando al Modelo Tradicional de herencia grecolatina y árabe, en su corriente gramatical, pero ahora se concretó en tres gramáticas que, a diferencia de la gramática de Merino, fueron más prácticas como el Compendio de gramática árabe vulgar (1908) del militar Fausto Santa Olalla que incluía un vocabulario hispano-árabe-militar para los militares que tenían que ejercer sus misiones en Marruecos. Otras dos gramáticas publicadas antes del Protectorado fueron el apéndice titulado «Nociones gramaticales del árabe vulgar de Marruecos» del arabista Antonio Almagro Cárdenas publicado en las Actas del Primer Congreso Español de Africanistas (1894) y extraído de su Compendio gramatical y léxico del árabe vulgar de Marruecos (1881) que no llegaría a publicarse (Gámez et al. 2000: 241-272) y Ensayo de gramática de árabe vulgar con aplicación al dialecto marroquí (1911) del militar Mariano Fernández Berbiela que se acercaba más a las nuevas gramáticas prácticas publicadas en 
otros países de Europa para la enseñanza de lenguas vivas.

El Modelo Gramatical y las obras de gramática quedarían ensombrecidos en esta etapa precolonial por la llegada de un método nuevo: el Método de Gramática y Traducción, puesto de moda en toda Europa por el lingüista alemán Heinrich G. Ollendorff y adaptado al árabe marroquí en España por el franciscano José María Lerchundi, autor de Rudimentos del árabe vulgar que se habla en el Imperio de Marruecos (1872). Rudimentos (Moscoso 2012a: 279292) se seguía basando en criterios gramaticales, aquí no hay muchas diferencias con respectos a las gramáticas tradicionales, pero, incluía como novedad la simplificación de los enunciados gramaticales y la inclusión de vocabularios y ejercicios para la traducción directa o inversa de frases. Aunque el manual de Lerchundi fue el más afamado de su tipo para facilitar el aprendizaje del árabe marroquí al misionero, al empleado, al industrial o al comerciante español, dos manuales más siguieron la estela de Rudimentos: Método práctico para hablar el árabe marroquí precedido de un silabario práctico de lectura y escritura al fin algunos apuntes geográficos (1908) del profesor Rafael Arévalo y Lecciones de árabe marroquí (1911) del profesor Pelayo Vizuete.

Y para quienes la gramática podía suponer una dificultad o no disponían de un profesor, el Modelo Tradicional de herencia grecolatina, en su corriente conversacional, basado en la creencia de que el árabe marroquí debía aprenderse conversando a partir de materiales extraídos de la propia realidad lingüística de Marruecos, supuso una alternativa eficaz a la enseñanza gramatical y permitió aprender, también, la cultura de los marroquíes. Este Modelo Conversacional se concretó en seis libros de diálogos cargados de vocabularios alfabéticos o temáticos, frases funcionales, diálogos y textos, relativos a la vida cotidiana o el conocimiento general de Marruecos. El primer librito de diálogos se publicó en forma de apéndice bajo el título «Voces y frases más necesarias para darse a entender en árabe» del arabista Serafín Estébanez Calderón que se incluyó en su Manual del oficial en Marruecos (1844). El apéndice incluía una breve descripción del árabe marroquí, un vocabulario árabe según el dialecto de Marruecos y algo más de una treintena de frases funcionales para uso militar escritas en transcripción figurada y acompañadas de su correspondiente traducción al español. Otros libritos de diálogos fueron el «Vocabulario de árabe según el dialecto marroquí» (1859) del africanista Manuel Torrijos, Manual del lenguaje vulgar de los moros de la Riff (1859) del militar Juan Albino (Moscoso 
2010, 121-140), Diálogos españoles-árabes o guía de la conversación Mogharbí (1860) del militar Pedro María del Castillo y Olivas (Moscoso 2012b, 109-129), Guía de la conversación española-arabe marroquí (1901) del intérprete Reginaldo Ruiz de Orsatti y El español en Marruecos. Método sencillísimo y práctico para hablar el árabe marroquí por medio de la pronunciación figurada (1906) del profesor Rafael Arévalo.

\section{La reorganización de la enseñanza del árabe marroquí en tiempos del Protectorado}

La instauración de un doble Protectorado franco-español en Marruecos entre 1912 y 1956 permitió que países como España y Francia (Moscoso 2014: 1-26) se erigieran como los máximos representantes de la enseñanza del árabe marroquí en Europa. En España, la enseñanza del árabe marroquí se reorganizó en una tercera etapa que abarca los más de cuarenta años que duró el Protectorado. Entonces, España hizo resurgir los vínculos históricos con Marruecos y la «hermandad hispano-marroquí» por cuestiones políticas y comerciales, convirtiéndose el árabe marroquí en un medio para defender y proteger los intereses de España en la Zona del Protectorado. El árabe marroquí se enseñó entonces en nuevos centros como la Escuela de Estudios Árabes de Granada, la Academia de Árabe y Beréber de Tetuán o la red de escuelas españolas creadas en la zona del Protectorado. Se intentó así formar a truchimanes del Servicio y del Cuerpo de Interpretación de Árabe y Bereber, a funcionarios de la nueva Administración colonial o a los niños españoles residentes en la Zona del Protectorado. Se publicaron, también, veintidós manuales de árabe marroquí gracias, especialmente, a los truchimanes, y en menor medida a militares o arabistas para contribuir así a «acción civilizadora» de España en Marruecos (Moscoso 2012c: 183-220).

\subsection{El Método Directo, el Método Ecléctico y el Modelo Conversacional}

Durante la etapa colonial el árabe marroquí se adaptó a las novedades metodológicas del llamado Método Directo (Sánchez 2009: 50-64), un método fraguado en Europa a finales del siglo XIX pero que vivió su mayor apogeo en la primera mitad del siglo XX en países como Francia o Alemania y, también, en Estados Unidos donde M. Berlitz popularizó la nueva metodología. El Método Directo había surgido como reacción al Método de Gramática y Traducción, gracias a las ideas introducidas por el llamado «Movimiento de Reforma», y se 
basó en una enseñanza natural, como aprenden los niños, inductiva y asociada a la imagen quedando la gramática y el uso de la lengua materna en un segundo plano. Este método natural se aplicó, con un cierto rigor, en cuatro manuales de árabe marroquí, siendo el primero de su tipo El soldado indígena: cartilla de lectura y escritura español y árabe. Libro primero (1916) del militar José Góngora y Rodríguez. Esta cartilla estuvo destinada a la formación en lengua castellana de los Regulares Indígenas pero, también, estaba diseñada para enseñar árabe marroquí a los europeos. Dividida en tres partes, la cartilla incluía información para el buen uso del manual por parte del profesor y contenía treinta lecciones breves que se iniciaban con una ilustración y ejercicios de lectura y escritura de frases breves. Por último, al final de manual se insertaban dos textos relativos a la historia de España y un vocabulario temático sobre la numeración. En adelante, se publicaron tres manuales más bajo el Método Directo: Método graduado de enseñanza por la imagen sin caracteres árabes adaptado al castellano. Conforme al sistema de las escuelas establecidas en Argel (1918) anónimo, Cartilla escolar de árabe. Primera parte (1950) y Cartilla escolar de árabe. Segunda parte [1950], ambas del intérprete José Aragón Cañizares.

Sin embargo, las críticas al Método Directo en España y en otros países de Europa por prescindir de la gramática como elemento sistematizador, dio lugar a la aparición de un Método Ecléctico, que no fue ni un claro representante del Método de Gramática y Traducción ni del Método Directo. De esta forma, se llegó a publicar once manuales de árabe marroquí de este tipo en España y que dieron continuidad al Método de Lerchundi hasta bien entrado el siglo XX. El primero de los manuales adaptados a este método mixto fue el Método AIS teórico-practico para la enseñanza del idioma árabe (1931) del intérprete Antonio Iglesias Seisdedos. Este manual, con prólogo de Rafael Arévalo, estuvo destinado a africanistas de muy diversos ámbitos como maestros, médicos o funcionarios civiles y contenía treinta diálogos bilingües en transcripción figurada y español sobre temas relativos a la vida cotidiana (en el colegio, en la calle, de viaje...), además, incluía nociones gramaticales sobre el alfabeto, el verbo, el nombre y la partícula que se ejercitaban mediante vocabularios y ejercicios de traducción directa o inversa. Otros manuales mixtos fueron Nociones de árabe vulgar. Para las escuelas de la Zona del Protectorado de España en Marruecos. Primer curso (1939) de los intérpretes Abderrahim Yebbur y Musa Abbud, Árabe dialectal marroquí. Primera parte (1944) y Árabe 
dialectal marroquí. Segunda parte (194?) del intérprete José Aragón Cañizares, Árabe marroquí. Tercera parte (s.a.) del intérprete Rafael Olmo Villafranca, Método P.P. para el rápido aprendizaje del árabe vulgar marroquí (1944) del intérprete Ginés Peregrín Peregrín, Método de árabe dialectal marroquí. Libro I y Libro II (s.a.) del profesor Fernando Valderrama, Árabe dialectal marroquí. Primer curso (1954) y Árabe dialectal marroquí. Segundo curso (1955) de los profesores Bonifacio Gómez, Abderrahim Yebbur Oddi y José Aragón Cañizares, y por último, se publicaba Método de árabe vulgar (1957) de María Valenzuela de Mulero, el único manual publicado por una mujer hasta entonces.

No faltaron tampoco quienes seguían apostando por el Modelo Conversacional. La publicación de siete libritos de diálogos que, con pocas novedades con respecto a los libros que se publicaron en vísperas del Protectorado, siguieron incluyendo vocabularios, frases funcionales, diálogos y textos para la práctica del idioma y el conocimiento general de Marruecos. El primero de su tipo fue la Guía manual de la conversación marroquí (1914) del arabista Ángel Muñoz Bosque, profesor del Centro Comercial de Madrid. Su manual tenía como finalidad poner al alcance de los africanistas el conocimiento práctico del árabe marroquí e incluía un buen número de frases funcionales para uso en la vida social («frases para rogar, pedir u ofrecer; frases para consentir, negar o excusarse; frases comerciales...»), así como también ofrecía «refranes y proverbios árabes» y una «lista con algunos nombres propios de personas, poblaciones, ríos, etc.». Otros libritos de diálogos publicados en adelante fueron Método práctico para el estudio del árabe hablado (1919) y La clave de la conversación hispano-árabe. Método fácil para aprender a hablar el árabe vulgar sin profesor por medio de la pronunciación figurada (1920) del intérprete Fermín Villalta y Llamas; Árabe vulgar al alcance de todos (pronunciación figurada) (1923) del funcionario de telégrafos Juan Alcántara Ruiz, Modelos de conversaciones árabes. Tratando variados y sugestivos asuntos de la vida práctica marroquí con pronunciación figurada, completa y sencilla y caracteres árabes, explicaciones gramaticales, descripción de usos, costumbres, creencias, etc., y lo más interesante de derecho musulmán (1924) del militar Ricardo Navas de Alda, Diálogos hispano-marroquíes: recopilación del Método A.I.S (1931) del intérprete Antonio Iglesias Seisdedos y Manual de la conversación de árabe vulgar marroquí. Método práctico y sencillo para iniciarse en el estudio del árabe vulgar (1940) del intérprete José Linares Rubio. 


\section{Nuevas perspectivas para la enseñanza del árabe marroquí tras el fin del Protectorado}

Tras la retirada de Francia y España de Marruecos en 1956 la enseñanza del árabe marroquí seguiría interesando en Europa por cuestiones académicas y migratorias. En España, la enseñanza del árabe marroquí cayó en el olvido tras el fin del Protectorado, pero la actividad volvería a reiniciarse a principios de los años 80 del siglo XX dando paso a una cuarta etapa que se prolonga hasta nuestros días. En este contexto comenzaron a publicarse interesantes investigaciones desde del ámbito académico español sobre dialectología, didáctica y pedagogía, que se sumaban a las ya realizadas en otros países como Inglaterra o Francia y Estados Unidos. Dichas investigaciones han contribuido a refinar notablemente el concepto sobre la naturaleza del árabe marroquí y, por ende, sobre su enseñanza y aprendizaje. Esto contribuyó a que la enseñanza de variedades vernáculas árabes, como el árabe marroquí, se fueran incluyendo en algunos programas de los hoy llamados Grados de Estudios Árabes e Islámicos o Grados en Lenguas Modernas de distintas universidades españolas y en los de otras Instituciones como la Escuela de Traductores de Toledo, Centro de Lengua Árabe de Casa Árabe en Madrid o Fundación Tres Culturas del Mediterráneo en Sevilla, por ejemplo, para satisfacer así las demandas de estudiantes, investigadores, funcionarios, voluntarios y otras personas atraídas por el árabe marroquí. Por otra parte, la enseñanza del árabe marroquí pretendió dar respuestas a las necesidades de la sociedad europea, receptora de importantes flujos migratorios procedentes de Marruecos en el transcurso del siglo XX. En el caso de España, de hecho, el árabe marroquí se ha ido convirtiendo en una de las lenguas más importantes de gran parte de la población originaria de Marruecos que vive en nuestro país y que ronda los 800.000 mil ciudadanos. Además, se han ido publicando entre 1982 y 2013 seis manuales de árabe marroquí para un público hispano-hablante que, alejados ya de un discurso colonial y de metodologías gramaticales, se han ido encaminando hacia los llamados Enfoques Comunicativos.

\subsection{Hacia los Enfoques Comunicativos}

La enseñanza del árabe marroquí desde finales del siglo XX inicia cambios relevantes gracias a las portaciones de la pedagogía, la psicología y la sociología a la enseñanza de lenguas. Aunque, en la actualidad, también hay que señalar los interesantes aportes que la neurociencia está haciendo al campo de la 
lingüística. De este modo, y con un relativo retraso con respecto a otras lenguas extranjeras, se mejoraron las deficiencias de métodos anteriores hasta que la enseñanza del árabe marroquí se ha ido adaptando a los actuales Enfoques Comunicativos.

El primer paso hacia el cambio en la enseñanza del árabe marroquí llegaría a comienzos de los años 80 del siglo XX, cuando se adaptó a los moldes del Método Situacional, un método originado de Estados Unidos durante el contexto de la Segunda Guerra Mundial, que se asentaba en la escuela estructuralista americana y en la psicología conductista y al que en Europa se añadió el concepto de situación (Sánchez 2009: 78-88). Bajo los principios de este método, el árabe marroquí fue entendido como un conjunto de hábitos lingüísticos que se daban en una situación determinada y que se adquirían mediante la repetición. Este método se concretó en dos manuales de árabe marroquí: Árabe vulgar. Contribución al estudio del árabe (1982) del intérprete y profesor de la UNED Manuel Martínez Martín, que incluía 51 temas de conversación y sus correspondientes grabaciones en casete, y Vamos a Fez: método de árabe dialectal marroquí (1987) del profesor del Centro Cultural de Fez, Mohammed Amrani (1987), que incluía diálogos basados en situaciones de la vida cotidiana y ejercicios de corte estructural (drills).

En adelante, el árabe marroquí se fue adaptando, tímidamente, a la enseñanza comunicativa de lenguas. Esta enseñanza ya se había puesto en marcha para otras lenguas extranjeras hacia los años 70 del siglo XX a través del llamado Enfoque Nocio-Funcional, resultado del Proyecto del Consejo de Europa denominado Nivel Umbral (Sánchez 2009: 95-128). Este enfoque se mejoraría en los años 90 concretándose en el llamado el Enfoque por Tareas que, en buena medida, se corresponde con el actual Enfoque Orientado a la Acción (EOA) propuesto por el MCER (Robles 2019: 1-17). Bajo estos Enfoques Comunicativos, el árabe marroquí se entiende como un instrumento para la comunicación y la realización de tareas en contextos de interacción mediante el desarrollo de competencias generales y comunicativas y el uso de estrategias. Y bajo estos criterios es como se diseña el método ¡Habla árabe marroquí! (2003) de la arabista Bárbara Herrero Muñoz-Cobo, un manual para principiantes autodidactas, que se acompaña de material de audio en formato CD. El manual se organiza en torno a siete unidades didácticas relacionadas con situaciones relativas a la vida cotidiana («Me llamo 'Ali, Qué hiciste ayer, iHola Fátima!...») e incluye contenidos lingüísticos (fonético-fonológicos y 
ortoépicos, gramaticales y léxicos) y contenidos extralingüísticos (comunicativo-pragmáticos y socio-culturales). Para ello, la autora propone diálogos, incluye ilustraciones, audios y actividades. Otros manuales que se publican en adelante son el Curso de árabe marroquí del arabista Francisco Moscoso (2006), que incluye material en formato CD; el manual Hablemos darija (2010) de la escritora $\mathbf{M}^{\mathrm{a}}$ Isabel Bassols y el manual Bchuia bchuiya (2013) de los profesores Francisco Moscoso, Nadi Hamdi Nouaouri y Óscar Rodríguez, este último, el único publicado en color, adaptado a los principios del MCER para un nivel A1 y acompañado no solo de audios sino también de vídeos. Además, cabe añadir la publicación de dos libritos de diálogos: la Guía de conversación en árabe magrebí (2005) de Adil Moustaoui y Radouane Zidi y Guía Marroquí para el viajero (2009), dos manuales que siguen dando continuidad al Modelo Conversacional hasta nuestros días.

Hoy la enseñanza del árabe marroquí en España sigue planteando nuevos retos metodológicos para dar respuesta a las demandas de la sociedad española en distintos ámbitos y contextos. Muchos de estos retos se han ido abordando en los siete Congresos de árabe marroquí celebrados entre 2006 y 2019 en distintas Universidades e instituciones españolas, como Casa Árabe de Madrid, que han dado espacio y voz a docentes e investigadores españoles y extranjeros. Igualmente, se han publicado algunas investigaciones que muestran las experiencias piloto que se están llevando a cabo en algunas universidades españolas que vienen a mejorar la práctica docente.

Algunas propuestas metodológicas en torno a la enseñanza del árabe marroquí apuntan hacia enfoques, métodos o modelos pedagógicos compatibles, a su vez, con los principios del MCER. El Aprendizaje Basado en Problemas (ABP) es una metodología apta para la enseñanza en la educación superior y con ella los profesores puede planificar, organizar y ejecutar una clase de una manera determinada para que adolescentes y adultos adquieran conocimientos que luego puedan aplicar a la resolución de problemas reales o simulados (Porlán 2017). Esta metodología adaptada a los principios del EOA propuesto por el MCER se está aplicando para la enseñanza del árabe marroquí desde el curso 17-18 en el Grado de Estudios Árabes e Islámicos de la Universidad de Sevilla (Salgado 2018: 714-734). Igualmente, existen modelos pedagógicos y técnicas de aprendizaje que también pueden ponerse en práctica para enseñar árabe marroquí, si bien, exigen la creación de manuales y otros materiales didácticos específicos, como el Flipped Clasroom, en el que los materiales 
educativos primarios se estudian en casa y luego se desarrollan tareas o proyectos en el aula, y la «Gamificación», que parte de técnicas de aprendizaje que permiten la integración de mecánicas y dinámicas propias de juegos o videojuegos para la enseñanza de lenguas.

Otras propuestas ponen de manifiesto las limitaciones del MCER cuando se aplica a la lengua árabe, pues, aunque el Marco contempla el bilingüismo y el contacto entre lenguas, no habla de diglosia. En efecto, el término lengua árabe, es amplio y complejo y hace referencia a las dos variedades de árabe que existen en la actualidad: el árabe normativo y las distintas variedades vernáculas de cada país arabófono, caso del árabe marroquí en Marruecos, las cuales tienen usos y funciones diferentes pudiéndose producir, además, un continuиm lingüístico entre ambos registros. Esta naturaleza diglósica de la lengua árabe, ha suscitado el interés de algunos docentes por el llamado Enfoque Integral, propuesto por el profesor Munther A. Younes (2015), que aboga por la enseñanza simultánea del árabe normativo y el árabe dialectal. Este Enfoque ha sido puesto en práctica por el profesor Antonio Giménez Reíllo en el Grado de Traducción e Interpretación de la Universidad de Murcia para la enseñanza del árabe marroquí desde 2015. En esta línea, la profesora Victoria Aguilar (2014: 305-324), partiendo de la experiencia lingüística en Cataluña y utilizando algunos trabajos de etnolingüística, ha hecho una propuesta para la enseñanza del árabe moderno y marroquí bajo el llamado Enfoque Integrado en la Universidad de Murcia, que contempla la enseñanza del árabe normativo y el árabe marroquí mediante dos programas diferenciados. Otras experiencias se orientan hacia Aprendizaje Integrado de Contenidos y Lenguas Extranjeras (AICLE), propuesto por David Marsh (1994), que consiste en enseñar de forma integrada contenidos y lengua. Esta propuesta ya fue puesta en práctica por el profesor Adil Moustaoui (2012: 225-234) en la Universidad Complutense de Madrid contemplando la enseñanza del árabe normativo y dialectal así como también las directrices del MCER y de The Islamic Educational Scientific and Cultural Organization (IESCO).

\section{Conclusiones}

En este artículo hemos mostrado la evolución metodológica de la enseñanza del árabe marroquí en nuestro país desde finales del siglo XVIII hasta la actualidad. Esta evolución metodológica ha sido similar a la de otras lenguas extranjeras de importancia equiparable, y quienes se dedicaron a ella han 
utilizado las dos tendencias tradicionalmente usadas en la enseñanza de lenguas en Europa, la gramatical y la conversacional, si bien, la gramatical arraigó con más fuerza en la enseñanza reglada hasta bien entrado el siglo XX por ser la gramática un elemento sistematizador, fácil de enseñar y cómodo para evaluar (Martín 2012: 37-70). Es por ello que hoy contamos con escasos manuales de árabe marroquí, todos ellos para principiantes, que se adapten a los Enfoques Comunicativos y solo uno se adapta con rigor a los principios del MCER para un nivel A1.

A tenor de las nuevas metodologías en auge para la enseñanza de lenguas y las experiencias de algunas universidades españolas, donde se aplican otros enfoques compatibles con los principios del MCER, como el Aprendizaje Basado en Problemas o el Enfoque Integral, la enseñanza del árabe marroquí sigue evolucionando desde el punto de vista metodológico. Sigue siendo necesario, sin embargo, seguir publicando investigaciones que ayuden a compartir experiencias y establecer conclusiones para continuar con la ardua labor de publicar nuevos manuales didácticos. En este sentido, aunque las metodologías y los manuales no son del todo suficientes en la enseñanza de lenguas, pues influyen otros factores relacionados con el docente, el discente o el contexto, contribuyen, sin embargo, a mejorar los procedimientos utilizados hasta el momento y a seguir trabajando para unificar criterios en torno a la enseñanza y el aprendizaje del árabe marroquí en el futuro.

\section{BIBLIOGRAFÍA}

AGUILAR, Victoria. 2014. «Enseñanza conjunta del árabe moderno normativo y el árabe marroquí». En Paula Santillán Grimm, Luis Miguel Pérez Cañada y Francisco Moscoso García (eds.). Árabe marroquí: de la oralidad a la enseñanza. Cuenca, Ediciones Castilla-La Mancha, pp. 305324.

AGUILAR, Victoria \& BOUHRASS, Asma. 2010. حجيتك، ماجيتك. Aprender marroquí en España, del XVIII al XXI». En: Bárbara Herrero MuñozCobo, Luis Miguel Péprez Cañada, Mercedes Aragón Huerta, Francisco Moscoso García (eds.). IV Congreso de Árabe marroquí: más allá de la 
oralidad. Almería, Editorial Universidad de Almería, pp. 163-186.

ARIAS TORRES, Juan Pablo. 1995. «Africanismo en primera persona: los métodos españoles de árabe coloquial marroquí», en Universidad de Granada (ed.). En: Homenaje al profesor José María Fórneas Besteiro. Granada, Universidad de Granada, pp. 321-336.

ARIAS TORRES, Juan Pablo \& PEÑA MARTÍN, Salvador. 1995. «Manuales españoles de coloquial marroquí». En: Carmelo Pérez y Caridad Ruíz (eds.). El Magreb. Coordenadas socioculturales. Granada, Universidad de Granada, pp. 11-38.

FERIA GARCÍA, Manuel C. 2005. «El tratado hispano-marroquí de amistad y comercio de 1767 en el punto de mira del Traductor (I). Contextualización histórica: encuentro y desencuentros». Sendebar 16, pp. 3-26, $<$ http://revistaseug.ugr.es/index.php/sendebar/article/view/1044> [24 de octubre de 2019].

GÁMEZ ROVIRA, María, MOSCOSO GARCÍA, Francisco \& RUIZ ROMAN, Lucía. 2000-2001. «Una gramática y un léxico de árabe marroquí escritos por Antonio Almagro». Al-Andalus-Magreb 8-9, pp. 241-272.

GIRARD, Aurélien. 2013. «Les manuels de langue arabe en usage en France à la fin de l'Ancien Régime». En: Sylvette Larzul \& Alain Messaoudi (dirs.). Manuels d'arabe d'hier et d'aujourd'hui. France et Magreb, XIX ${ }^{e}$ $X X I^{e}$ siècle. Paris, Bibliotèque Nationale de France, pp. 12-26, <https://books.openedition.org/editionsbnf/259>, [24 de octubre de 2019].

GIMÉNEZ REÍLLO, Antonio. Blog Anís del Moro. $<$ http://anisdelmoro.blogspot.com> [24 de octubre de 2019].

GÓMEZ FONT, Alberto. 1995. «Obras en español para el aprendizaje del árabe dialectal marroquí». Algarabía 5, pp. 18-23.

JUSTEL CALABOZO, Braulio. 1991. El toledano Patricio de la Torre. Monje Escurialense. Arabista y Vicecónsul en Tánger. Madrid, Ediciones Escurialenses, Real Monasterio de El Escorial.

Marco Común Europeo de Referencia para las Lenguas: Aprendizaje, Enseñanza, Evaluación. 2001. Madrid, Consejo de Europa, Instituto Cervantes, Ministerio de Educación, Anaya.+ $<$ https://cvc.cervantes.es/ensenanza/biblioteca ele/marco/> [24 de octubre de 2019].

LARZUL, Sylvette. 2013. «Les Manuels de lengua arabe des débuts de l'Algérie coloniale (1830-1871)». En Sylvette Larzul \& Alain Messaoudi (dirs.). 
Manuels d'arabe d'hier et d'aujourd'hui: France et Maghreb, XIX $X^{e}-X X I^{e}$ siècle. Paris, Éditions de la Bibliothèque Nationale de France, pp. 54-78. <http://books.openedition.org/editionsbnf/244 > [24 de octubre de 2019].

MASRH, David. 1994. Bilingual Education \& Content and Language Integrated Learning. International Association for Cross-cultural Communication, Language Teaching in the Member State of the European Union (Lingua). Paris, Université Paris-Sorbonne.

MARTÍN Tormo, Vicente. 2012. «Metodología pedagógica e investigación en el campo de la enseñanza y aprendizaje del árabe como segunda lengua: elementos para un estado de la cuestión». Miscelanea de Estudios Árabes y Hebraicos. Sección árabe-islam 61, pp. 37-60.

MOSCOSO GARCÍA, Francisco. 2014. «Libros para el estudio del árabe marroquí escritos durante el Protectorado francés en Marruecos». Revista de Estudios Internacionales Mediterráneos 16, pp. 1-26.

<https://revistas.uam.es/index.php/reim/article/view/937/3719> [24 de octubre de 2019].

MOSCOSO GARCÍA, Francisco. 2012a. «Los inicios del estudio del árabe marroquí. Los Rudimentos del P. Lerchundi». En: Ana Agud (coord.). Séptimo Centenario de los Estudios Orientales en Salamanca. Salamanca, Universidad de Salamanca, pp. 279-292.

MOSCOSO GARCÍA, Francisco. 2012b. «El interés por el estudio del árabe vulgar generado en torno a la Guerra de África. El plagio de un patriota, Pedro María del Castillo y Olivas». Anaquel de Estudios Árabes 23, pp. 109-129.

MOSCOSO GARCÍA, Francisco. 2012c. «Árabe marroquí: vulgar y dialectal. El interés por su aprendizaje y su metodología de estudio durante el Protectorado». Revista de Estudios Internacionales Mediterráneos 12, pp. 183-220.

<https://revistas.uam.es/index.php/reim/article/view/879 >, [24 de octubre de 2019].

MOSCOSO GARCÍA, Francisco 2010. «Un manual para aprender el árabe marroquí escrito en el Peñón de Vélez de la Gomera». Al-Andalus-Magreb 17, pp. 121-140.

MOSCOSO GARCÍA, Francisco. 2008. «El estudio del árabe marroquí en España durante el siglo XIX. La obra de Manuel Bacas Merino». Miscelánea de Estudios Árabes y Hebraicos. Sección árabe-islam 57, pp. 
269-293.

MOUSTAOUI, Adil y ZIDI, Radouane, Guía de conversación en árabe magrebí. Guía esencial para el viajero, Espasa Calpe, Madrid, 2005.

MOUSTAOUI SRHIR, Adil. 2012. «El enfoque integrado en la enseñanza del árabe como lengua extranjera y de contenidos "Sociales" en la Universidad española: consideraciones sociolingüísticas y desafíos didácticos. En: Ruth Jiménez Berrio, Felipe Llamas Saiz, Concepción Martínez Pasamar \& Cristina Taberno Sala (eds.). Teaching approaches to CLIC/Propuestas docentes en AICLE. Pamplona, Servicio de Publicaciones de la Universidad de Navarra, pp. 225-234.

PORLÁN, Rafael. 2017. Enseñanza universitaria. Cómo mejorarla. Madrid, Morata.

ROBLES Ávila, Sara. 2019. «A vueltas con el enfoque orientado a la acción: leyendo las nuevas aportaciones desde el volumen complementario del MCER (2017)». Álabe. Revista de la Red de Universidades Lectoras 19, <http://revistaalabe.com/index/alabe/article/view/498 > [24 de octubre de 2019].

SANCHEZ PÉREZ. Aquilino. 2009. La enseñanza de idiomas en los últimos cien años: métodos y enfoques. Madrid, SGEL.

SÁNCHEZ PÉREZ, Aquilino. 1992. Aquilino Sanchez. Historia de la enseñanza del español como lengua extranjera. Madrid, SGEL.

SALGADO Suárez, Rosa. 2018. «Una contribución para la innovación docente en la enseñanza del árabe marroquí como lengua extranjera (EáMLE)». Jornadas de Formación e Innovación Docente del Profesorado, pp. 714734. 〈http://institucional.us.es/revistas/JDU/Salgado_Suarez_Rosa.pdf> [24 de octubre de 2019].

SALGADO Suárez, Rosa. 2017. «Las relaciones hispano-marroquíes y la enseñanza del árabe marroquí. El legado didáctico de los arabistas». En: Damián A. González, Manuel Ortíz Heras \& Juan Sisinio Pérez Garzón. La historia lost in translation? Actas del XIII Congreso de la Asociación de Historia Contemporánea. Cuenca, Universidad de Castilla-La Mancha, pp. 2623-2637.

<https://publicaciones.uclm.es/la-historia-lost-in-translation/> $\left[\begin{array}{ll}24 & \text { de }\end{array}\right.$ octubre de 2019].

VICENTE, Ángeles. 2008. «Génesis y clasificación de los dialectos neoárabes». En: Federico Corriente y Ángeles Vicente (eds.). Manual de dialectología 
neoárabe. Zaragoza, Instituto de Estudios Islámicos y del Oriente Próximo, pp. 19-67.

YOUNES, Mounther. 2015. The Integrated Approach to Arabic Instruction, New York, Routledge.

ZWARTJES, Otto. 1993. «El artículo en las gramáticas pioneras de Antonio de Nebrija y Pedro de Alcalá y las gramáticas grecolatinas». En Maxim P.A.M. Kerkhof, Hugo Schepper y Otto Zwartjes (eds.). Diálogos hispánicos, España: ¿Ruptura 1492? 11. Amsterdam-Atlanta, G.A: Rodopi, pp. 261-288.

\section{MANUALES DE ÁRABE MARROQUÍ PUBLICADOS EN ESPAÑOL}

ALBINO, Juan. 1859. Manual del lenguaje vulgar de los moros de la-Riff. Cádiz, Imprenta de la Revista Médica.

ALCÁNTARA RUIZ, Juan. 1923. Árabe vulgar al alcance de todos (pronunciación figurada). Tetuán, Imprenta Martínez.

ALMAGRO CÁRDENAS, Antonio. 1894. «Nociones gramaticales del árabe vulgar de Marruecos». En: Actas y Memorias del Primer Congreso Español de Africanistas celebrado en Granada con motivo y en conmemoración del IV Centenario del Descubrimiento de América. Granada, Tipografía del Hospital de Santa Ana, pp. 85-108.

AMRANI, Mohammed. 1987. Vamos a Fez: método de árabe dialectal marroquí ( $1^{o}$ nivel). Fez, Centro Cultural Español de Fez (2 fasc.).

ARAGÓN CAÑIZARES, José. 1950. Cartilla escolar de árabe. Primera parte. Tetuán, Editora Marroquí.

ARAGÓN CAÑIZARES. 1950. José, Cartilla escolar de árabe. Segunda parte. Tetuán, Editora Marroquí.

ARAGÓN CAÑIZARES, José. 1944. Árabe dialectal Marroquí. Primera parte. Larache, Editora Marroquí.

ARAGÓN CAÑIZARES, José [194-?]: Árabe dialectal Marroquí. Segunda parte. Larache, Editora Marroquí.

ARÉVALO, Rafael. 1908. Método práctico para hablar el árabe-marroquí precedido de un silabario práctico de lectura y escritura al fin algunos 
apuntes geográficos. Tánger, Librería española.

ARÉVALO, Rafael. 1906. El español en Marruecos. Método sencillísimo y práctico para hablar el árabe marroquí por medio de la pronunciación figurada. Tánger, Librería española.

BACAS MERINO, Manuel. 1807. Compendio Gramatical para aprender la lengua arábiga, así sabia como vulgar. Madrid, Imprenta Sancha.

BASSOLS, Isabel. 2010. Hablemos darija (Árabe marroquí) y Hablemos español. Tánger, Editions Slaiki Frères.

CASTILLO Y OLIVAS, Pedro María del. 1860. Diálogos españoles-árabes o guía de la conversación Mogharbí. Madrid, Imprenta M. Galiano.

ESTÉBANEZ CALDERÓN, Serafín. 1844. «Voces y frases más necesarias para darse á entender en árabe». En: Manual del oficial en Marruecos o cuadro geográfico, estadístico, histórico, político y militar de aquel imperio». Madrid, Imprenta de Ignacio Boix, pp. 321-331.

FERNÁNDEZ BERBIELA, Mariano. 1911. Ensayo de gramática de árabe vulgar con aplicación al dialecto marroquí. Ceuta-Tetuán, Max L. Tornow y Cía., Frankfurt a.M.

GÓMEZ MARTÍNEZ, Bonifacio, YEBBUR ODDI, Abderrahim \& ARAGÓN CAÑIZARES, José. 1955. Árabe dialectal marroquí. Segundo curso. Tetuán, Editora Marroquí.

GÓMEZ MARTÍNEZ, Bonifacio, YEBBUR ODDI, Abderrahim \& ARAGÓN CAÑIZARES, José. 1954. Árabe dialectal marroquí. Primer curso. Tetuán, Impresa Majzén.

GÓNGORA Y RODRÍQUEZ, José. 1916. El soldado indígena: cartilla de lectura y escritura español y árabe. Libro primero. Melilla, Imprenta y encuadernación «la Africana».

HERRERO MUÑOZ-COBO, Bárbara. 2003. ¡Habla árabe marroquí! Método para principiantes. Madrid, Ibersaf.

IGLESIAS SEISDEDOS, Antonio. 1931. Diálogos hispano-marroquíes: recopilación del Método A.I.S. Tetuán-Tánger, La Papelería Africana.

IGLESIAS SEISDEDOS, Antonio. 1931. Método A.I.S. Teórico-práctico para la enseñanza del idioma árabe. Tetuán-Tánger, Papelería Africana.

LERCHUNDI, José María de. 1872. Rudimentos del árabe vulgar que se habla en el imperio de Marruecos. Con numerosos ejercicios y temas aplicados a la teoría. Imprenta y estereotipia de M. Rivadeneryra [edición facsímil], con prólogo de Ramón Lourido Díaz. Madrid, AECI. 
LINARES RUBIO, José. 1949. Manual de la conversación de árabe vulgar marroquí. Método práctico y sencillo para iniciarse en el estudio del árabe vulgar. Larache.

MARTÍNEZ MARTÍN, Manuel. 1982. Árabe vulgar. Contribución al estudio del árabe. 51 temas de conversación grabados en «cassette» con sus correspondientes apuntes gramaticales. Melilla, Graficasa.

Método graduado de enseñanza por la imagen sin caracteres árabes adaptado al castellano. Conforme al sistema de las escuelas establecido en Argel. 1918. Tetuán, Editorial Hispano Africana.

MOSCOSO GARCÍA, Francisco, Nadi Hamdi NOUAOURI IZRELLI, y Óscar RODRÍGUEZ GARCÍA. 2013. بشوية بشوية《 Bchuiya bchuiya, Árabe marroqui». Almería, Albujaira.

MOSCOSO GARCÍA, Francisco. 2006. Curso de árabe Marroquí: diálogos, gramática, ejercicios, glosario. Cádiz, Universidad de Cádiz.

MUÑOZ BOSQUE, Ángel. 1914. Manual de la conversación marroquí. Madrid, Artes Gráficas.

NAVAS DE ALDA, Ricardo. 1924‥ Modelos de conversaciones árabes. Tratando variados y sugestivos asuntos de la vida práctica marroquí con pronunciación figurada, completa y sencilla y caracteres árabes, explicaciones gramaticales, descripción de usos, costumbres, creencias, etc., y lo más interesante de derecho musulmán. Larache, Casa editora. Establecimientos tipográficos "Goya".

OLMO VILLAFRANCA, Rafael (s.a.). Árabe marroquí. Tercera parte. Tetuán, Editora Marroquí.

PEREGRÍN PEREGRÍN, Ginés. 1944. Método P.P. para el rápido aprendizaje del árabe vulgar marroquí. Tetuán, Tipografía el-Mahdía.

RUIZ ORSATTI, Reginaldo (1901): Guía de la conversación española-árabe marroquí. Tánger, Imprenta Hispano-Arábiga de la Misión Católica.

SANTA OLALLA MILlET, Fausto. 1908. Compendio de gramática árabe vulgar y vocabulario hispano-árabe-militar. Tánger, Establecimiento tipográfico S. Benaioun.

TORRIJOS, Manuel. 1859. «Vocabulario árabe según el dialecto marroquí». El Imperio de Marruecos, su historia, topografía, estadística, religión, costumbres, industria, agricultura, artes, milicia, etc.». Madrid, Imprenta Eduardo Martínez, pp. 231-236.

VALDERRAMA MARTÍNEZ, Fernando (s.a.). Método de árabe dialectal 
marroquí. Libro II. Tetuán, Editora marroquí.

VALDERRAMA MARTÍNEZ, Fernando (s.a.). Método de árabe dialectal marroquí. Libro I. Tetuán, Editora marroquí.

VALENZUELA DE MULERO, María. 1957. Método de árabe vulgar. Tetuán, Editorial Cremades.

VILLALTA Y LLAMAS, Fermín. 1920. La clave de la conversación hispanoárabe. Método fácil para aprender a hablar el árabe vulgar sin profesor por medio de la pronunciación figurada. Ceuta, Taller Arturo Sierra.

VILLALTA Y LLAMAS, Fermín. 1919. Método práctico para el estudio del árabe hablado. Tetuán, Imprenta de la Viuda e Hijos de Francisco Vialá.

VIZUETE, Pelayo. 1911. Lecciones de árabe marroquí. Barcelona, Sucesores de Manuel Soler.

YEBBUR, Abderrahim \& ABBUD, Musa. 1939. Nociones de árabe vulgar. Para las Escuelas de la Zona de Protectorado de España en Marruecos. Primer curso. Tánger, Tipografía F. Erola, Tánger. 